\title{
Crystal structure of 1,3,5-tri-2-propyl-2,4-dioxohexahydro-1,3,5-triazine, $\mathrm{C}_{12} \mathrm{H}_{23} \mathrm{~N}_{3} \mathrm{O}_{2}$
}

\author{
K. O. Klepp ${ }^{*, \mathrm{I}}$ and H. Schmidt ${ }^{\mathrm{II}}$ \\ I Universität Linz. Abteilung für Allgemeine und Anorganische Chemie, Altenbergerstraße 69, A-4040 Linz, Austria \\ II Universität Linz. Institut für Chemische Technologie Organischer Stoffe, Altenbergerstraße 69. A-4040 Linz, Austria
}

Received August 14, 2002, accepted and available on-line October 2, 2002; CCDC-No. $1267 / 916$
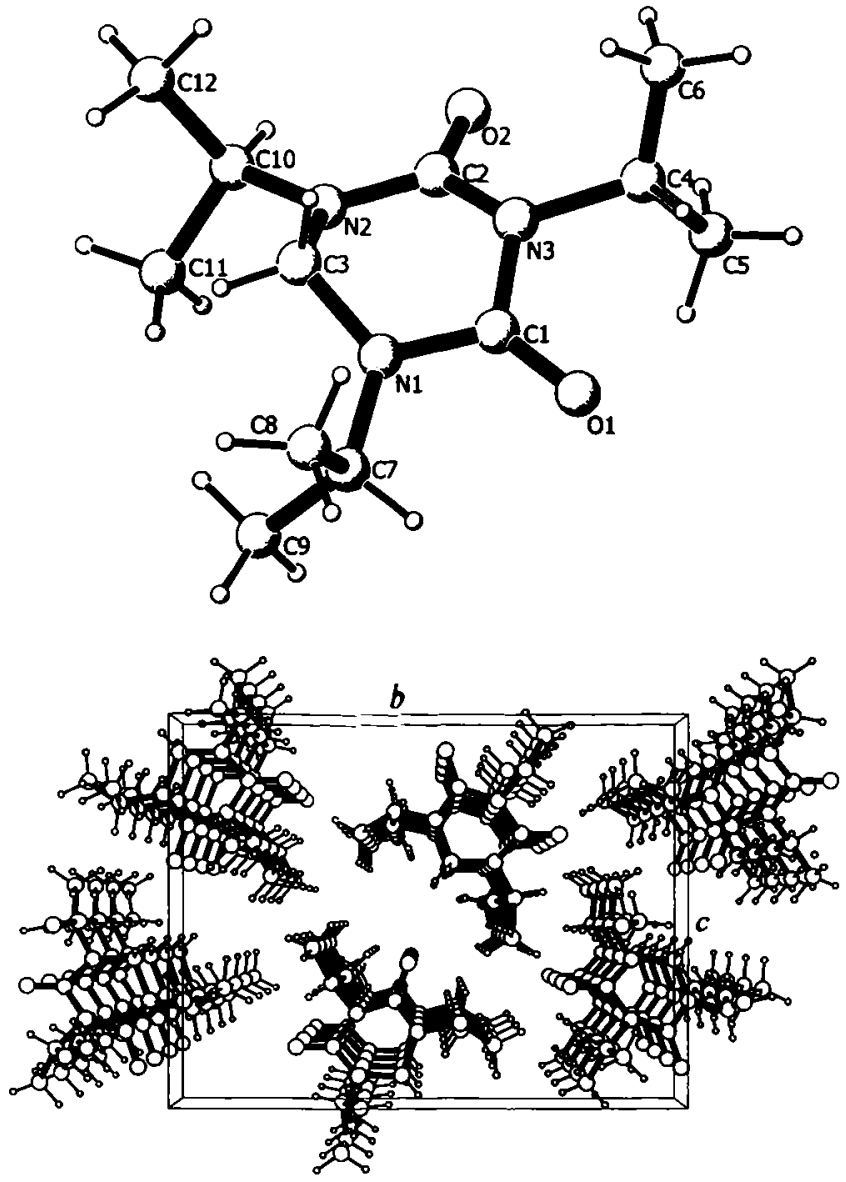

Abstract

$\mathrm{C}_{12} \mathrm{H}_{23} \mathrm{~N}_{3} \mathrm{O}_{2}$, orthorhombic, $P 2{ }_{1} 2_{1} 2_{1}$ (No. 19), $a=6.033(2) \AA$ $b=17.658(8) \AA, c=13.224(5) \AA, V=1408.8 \AA^{3}, Z=4$, $R_{\mathrm{gt}}(F)=0.046, w R_{\mathrm{obs}}\left(F^{2}\right)=0.048, T=294 \mathrm{~K}$.

\section{Source of material}

The title compound can be obtained by reacting 2,4-dioxohexahydro-1,3,5-triazine with 2-chlorpropane and powdered potassium hydroxide in anhydrous dimethylsulfoxide [1].

\section{Discussion}

The 1,3,5-tri-2-propyl-2,4-dioxohexahydro-1,3,5-triazine molecule is characterized by a puckered ring which adopts a boatlike conformation (upper figure). The atoms $\mathrm{C} 1, \mathrm{C} 2, \mathrm{~N} 1$ and $\mathrm{N} 2$ are in a virtually planar arrangement (mean deviation $0.008 \AA$ ) from which $\mathrm{C} 3$ and $\mathrm{N} 3$ are offset by $0.578 \AA$ and $0.305 \AA$, respectively. These values are distinctly larger than the corresponding displacements observed for 1,3,5-trimethyl-2,4-dioxohexahydro1,3,5-triazine [2]. In the crystal structure, the molecules are stacked in piles along [100] which in turn are arranged in a distorted hexagonal rod packing (lower figure).

Table 1. Data collection and handling.

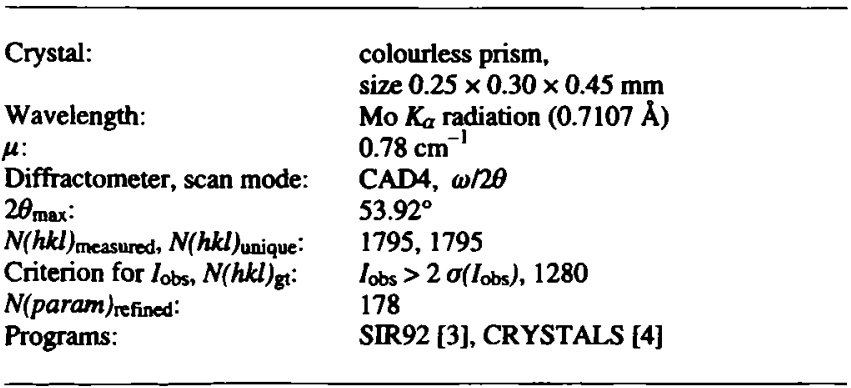

Table 2. Atomic coordinates and displacement parameters (in $\AA^{2}$ ).

\begin{tabular}{|c|c|c|c|c|c|}
\hline Atom & Site & $x$ & $y$ & $z$ & $U_{\text {iso }}$ \\
\hline $\mathbf{H}(\mathbf{l})$ & $4 a$ & $0.4684(5)$ & $0.0164(1)$ & $0.5742(2)$ & $0.067(1)$ \\
\hline$H(2)$ & $4 a$ & $0.2247(5)$ & $0.0385(1)$ & $0.5969(2)$ & 0.067 \\
\hline$H(6)$ & $4 a$ & $0.2011(6)$ & $-0.1153(2)$ & $0.7094(3)$ & $0.109(2)$ \\
\hline $\mathbf{H}(7)$ & $4 a$ & $0.3632(6)$ & $-0.1130(2)$ & $0.6184(3)$ & 0.109 \\
\hline$H(8)$ & $4 a$ & $0.4068(6)$ & $-0.1681(2)$ & $0.7076(3)$ & 0.109 \\
\hline$H(9)$ & $4 a$ & $0.4663(6)$ & $-0.0692(2)$ & $0.8094(3)$ & $0.085(1)$ \\
\hline$H(10)$ & $4 a$ & $0.2850(6)$ & $0.1725(2)$ & $0.4464(2)$ & $0.093(2)$ \\
\hline $\mathrm{H}(11)$ & $4 a$ & $0.5003(6)$ & $0.1755(2)$ & $0.3824(2)$ & $0.093(2)$ \\
\hline$H(12)$ & $4 a$ & $0.4087(6)$ & $0.0982(2)$ & $0.4192(2)$ & 0.093 \\
\hline$H(13)$ & $4 a$ & $0.7648(5)$ & $0.0715(2)$ & $0.5101(3)$ & $0.117(2)$ \\
\hline$H(14)$ & $4 a$ & $0.8540(5)$ & $0.1488(2)$ & $0.4720(3)$ & 0.117 \\
\hline$H(15)$ & $4 a$ & $0.8524(5)$ & $0.1308(2)$ & $0.5868(3)$ & 0.117 \\
\hline$H(16)$ & $4 a$ & $0.5690(5)$ & $0.2088(2)$ & $0.5475(2)$ & $0.073(1)$ \\
\hline H(17) & $4 a$ & $-0.2130(7)$ & $0.1377(3)$ & $0.7891(4)$ & $0.201(3)$ \\
\hline$H(18)$ & $4 a$ & $-0.1129(7)$ & $0.0977(3)$ & $0.8831(4)$ & 0.201 \\
\hline H(19) & $4 a$ & $-0.2129(7)$ & $0.1783(3)$ & $0.8931(4)$ & 0.201 \\
\hline$H(20)$ & $4 a$ & $0.3237(7)$ & $0.2382(2)$ & $0.8870(2)$ & $0.103(2)$ \\
\hline $\mathbf{H}(21)$ & $4 a$ & $0.1170(7)$ & $0.2371(2)$ & $0.9567(2)$ & 0.103 \\
\hline $\mathrm{H}(22)$ & $4 a$ & $0.2734(7)$ & $0.1674(2)$ & $0.9526(2)$ & 0.103 \\
\hline$H(23)$ & $4 a$ & $0.0413(6)$ & $0.2127(2)$ & $0.7929(3)$ & $0.112(2)$ \\
\hline$H(3)$ & $4 a$ & $0.7750(6)$ & -0.1139 (2) & $0.7281(3)$ & $0.138(2)$ \\
\hline H(4) & $4 a$ & $0.7405(6)$ & $-0.0554(2)$ & $0.6419(3)$ & 0.138 \\
\hline H(5) & $4 a$ & $0.8017(6)$ & $-0.0281(2)$ & $0.7499(3)$ & 0.138 \\
\hline
\end{tabular}

* Correspondence author (e-mail: kurt.klepp@jku.at) 
Table 3. Atomic coordinates and displacement parameters (in $\AA^{2}$ ).

\begin{tabular}{|c|c|c|c|c|c|c|c|c|c|c|}
\hline Atom & Site & $x$ & $y$ & $z$ & $U_{11}$ & $U_{22}$ & $U_{33}$ & $U_{12}$ & $U_{13}$ & $U_{23}$ \\
\hline$O(1)$ & $4 a$ & $0.2655(5)$ & $0.0341(1)$ & $0.8786(1)$ & $0.125(2)$ & $0.094(2)$ & $0.056(1)$ & $-0.015(2)$ & $0.017(2)$ & $0.002(1)$ \\
\hline $\mathrm{O}(2)$ & $4 a$ & $0.3247(4)$ & $0.2360(1)$ & $0.6821(2)$ & $0.096(2)$ & $0.055(1)$ & $0.084(1)$ & $0.008(1)$ & $0.001(1)$ & $-0.011(1)$ \\
\hline$N(1)$ & $4 a$ & $0.3926(5)$ & $0.0158(1)$ & $0.7197(2)$ & $0.082(2)$ & $0.054(1)$ & $0.053(1)$ & $0.006(1)$ & $0.004(1)$ & $0.001(1)$ \\
\hline$N(2)$ & $4 a$ & $0.4306(4)$ & $0.1224(1)$ & $0.6175(2)$ & $0.069(2)$ & $0.047(1)$ & $0.057(1)$ & $0.001(1)$ & $0.008(1)$ & $-0.003(1)$ \\
\hline $\mathbf{N}(3)$ & $4 a$ & $0.2161(4)$ & $0.1271(1)$ & $0.7611(2)$ & $0.057(1)$ & $0.059(1)$ & $0.061(1)$ & $-0.000(1)$ & $0.009(1)$ & $-0.017(1)$ \\
\hline$C(3)$ & $4 a$ & $0.3742(5)$ & $0.0436(1)$ & $0.6177(2)$ & $0.067(2)$ & $0.050(2)$ & $0.050(1)$ & $0.005(1)$ & $0.004(1)$ & $-0.005(1)$ \\
\hline$C(2)$ & $4 a$ & $0.3253(5)$ & $0.1670(2)$ & $0.6849(2)$ & $0.056(2)$ & $0.056(2)$ & $0.061(2)$ & $0.004(1)$ & $-0.005(2)$ & $-0.010(1)$ \\
\hline$C(1)$ & $4 a$ & $0.2909(5)$ & $0.0565(2)$ & $0.7930(2)$ & $0.069(2)$ & $0.066(2)$ & $0.055(1)$ & $-0.013(2)$ & $0.001(2)$ & $-0.007(1)$ \\
\hline$C(4)$ & $4 a$ & $0.0772(6)$ & $0.1715(2)$ & $0.8317(3)$ & $0.067(2)$ & $0.108(3)$ & $0.104(3)$ & $-0.003(2)$ & $0.022(2)$ & $-0.056(2)$ \\
\hline$C(5)$ & $4 a$ & $0.2096(7)$ & $0.2078(2)$ & $0.9140(2)$ & $0.100(3)$ & $0.087(2)$ & $0.070(2)$ & $-0.007(2)$ & $0.016(2)$ & $-0.028(2)$ \\
\hline$C(6)$ & $4 a$ & $-0.1327(7)$ & $0.1450(3)$ & $0.8502(4)$ & $0.074(3)$ & $0.249(6)$ & $0.180(5)$ & $-0.004(4)$ & $0.025(3)$ & $-0.130(5)$ \\
\hline$C(7)$ & $4 a$ & $0.4822(6)$ & $-0.0595(2)$ & $0.7395(3)$ & $0.083(2)$ & $0.060(2)$ & $0.069(2)$ & $0.009(2)$ & $-0.004(2)$ & $0.012(2)$ \\
\hline$C(8)$ & $4 a$ & $0.3504(6)$ & $-0.1199(2)$ & $0.6882(3)$ & $0.106(3)$ & $0.057(2)$ & $0.109(3)$ & $0.008(2)$ & $0.008(3)$ & $-0.001(2)$ \\
\hline C(9) & $4 a$ & $0.7215(6)$ & $-0.0648(2)$ & $0.7121(3)$ & $0.076(2)$ & $0.106(3)$ & $0.164(4)$ & $0.020(2)$ & $-0.009(3)$ & $0.026(3)$ \\
\hline$C(10)$ & $4 a$ & $0.5521(5)$ & $0.1564(2)$ & $0.5327(2)$ & $0.060(2)$ & $0.056(2)$ & $0.065(2)$ & $-0.009(1)$ & $0.004(2)$ & $0.004(1)$ \\
\hline$C(11)$ & $4 a$ & $0.7805(5)$ & $0.1242(2)$ & $0.5239(3)$ & $0.063(2)$ & $0.134(3)$ & $0.097(3)$ & $0.001(2)$ & $0.011(2)$ & $0.026(3)$ \\
\hline$C(12)$ & $4 a$ & $0.4243(6)$ & $0.1509(2)$ & $0.4363(2)$ & $0.081(2)$ & $0.082(2)$ & $0.069(2)$ & $-0.010(2)$ & $-0.006(2)$ & $0.016(2)$ \\
\hline
\end{tabular}

\section{References}

1. Schmidt, H.; Stähr, M.: Zum Reaktionsverhalten von 2,4-Dioxohexahydro-1,3,5-triazin. Monatsh. Chemie 128 (1997) 475-481.

2. Klepp, K. O.; Stähr, M.; Schmidt, H.: The crystal structure of 1,3,5trimethyl-2,4-dioxohexahydro-1,3,5-triazine, $\mathrm{C}_{6} \mathrm{H}_{11} \mathrm{~N}_{3} \mathrm{O}_{2}$. Z. Kristallogr. NCS 215 (2000) 147-148.
3. Altomare, G.; Cascarano, G.; Giaccovazzo, C.; Guagliardi, A.; Burla, M. C.; Polidori, G.: SIR92 - a program for automatic solution of crystal structures by direct methodes. J. Appl. Crystallogr. 27 (1994) 435-436.

4. Watkin, D. J.; Prout, C. K.; Carruthers, J. R.; Betteridge, P. W.: CRYSTALS, Chemical Crystallography Laboratory, Oxford, UK 1996. 